\title{
Chemically Triggered Formation of \\ Two-Dimensional Epitaxial Quantum Dot Superlattices
}

\author{
Willem Walravens, ${ }^{\dagger, \ddagger}$ Jonathan De Roo, ${ }^{\mathbb{I}, \dagger}$ Emile Drijvers, ${ }^{\dagger, \dagger}$ Stephanie ten \\ Brinck, ${ }^{\S}$ Eduardo Solano,\| Jolien Dendooven, "Christophe Detavernier, $\|, \ddagger$ Ivan \\ Infante, ${ }^{\S}$ and Zeger Hens*,†, \\ Physics and Chemistry of Nanostructures (PCN), Ghent University, Ghent, Belgium, \\ Center for Nano and Biophotonics, Ghent University, Ghent, Belgium, Sol-gel Center for \\ Research on Inorganic Powders and Thin films Synthesis (SCRiPTS), Ghent University, \\ Ghent, Belgium, Department of Theoretical Chemistry, Vrije Universiteit Amsterdam, \\ Amsterdam, The Netherlands, and Conformal Coating of Nanomaterials (CoCooN), Ghent \\ University, Ghent, Belgium \\ E-mail: zeger.hens@ugent.be
}

\begin{abstract}
Two dimensional superlattices of epitaxially connected quantum dots enable sizequantization effects to be combined with high charge carrier mobilities, an essential prerequisite for highly performing QD devices based on charge transport. Here, we

${ }^{*}$ To whom correspondence should be addressed

${ }^{\dagger} \mathrm{PCN}$, Ghent University

${ }^{\ddagger}$ Photonics group, Ghent University

"SCRiPTS, Ghent University

$\S$ VU Amsterdam

"CoCoon
\end{abstract}


demonstrate that surface active additives known to restore nanocrystal stoichiometry can trigger the formation of epitaxial superlattices of $\mathrm{PbSe}$ and $\mathrm{PbS}$ quantum dots. More specifically, we show that both chalcogen-adding (sodium sulfide) and lead oleate displacing (amines) additives induce small area epitaxial superlattices of PbSe quantum dots. In the latter case, the amine basicity is a sensitive handle to tune the superlattice symmetry, with strong and weak bases yielding pseudo-hexagonal or quasi-square lattices, respectively. Through density functional theory calculations and in-situ titrations monitored by nuclear magnetic resonance spectroscopy, we link this observation to the concomitantly different coordination enthalpy and ligand displacement potency of the amine. Next to that, an initial $\approx 10 \%$ reduction of the initial ligand density prior to monolayer formation and addition of a mild, lead oleate displacing chemical trigger such as aniline proved key to induce square superlattices with long range, $\mu \mathrm{m}^{2}$ order; an effect that is the more pronounced the larger the quantum dots. As the approach applies to $\mathrm{PbS}$ quantum dots as well, we conclude that it offers a reproducible and rational method for the formation of highly ordered epitaxial quantum dot superlattices.

Colloidal nanocrystals made by highly precise synthesis methods such as hot injection have been widely used as building blocks of self-assembled nanocrystal superlattices. ${ }^{1-5}$ Especially in the case of semiconductor nanocrystals or quantum dots (QDs), formation of highly involved binary or ternary superstructures has been demonstrated, ${ }^{6-10}$ the symmetry of which could be rationalized using hard sphere crystallization theory. ${ }^{10-13}$ Whereas this provides ample possibilities to combine different nanocrystals in a single, ordered crystal, only few studies have shown such an approach to result in metamaterials with new or enhanced properties. ${ }^{14-16}$ For one thing, this is due to the use of nanocrystal building blocks capped by long, organic ligands, which inevitably leads to electrically insulating nanocrystal solids. Therefore, opto-electronic devices such as transistors, ${ }^{17-19}$ solar cells ${ }^{20-23}$ or photodetectors, ${ }^{24-26}$ are based on disordered QD solids, where the interparticle distance is usually decreased by exchanging the long organic ligands with shorter organic or inor- 
ganic moieties. ${ }^{27-31}$ Although this makes for QD devices with ever increasing performance, carrier mobilities remain well below $10 \mathrm{~cm}^{2} \mathrm{~V}^{-1} \mathrm{~s}^{-1}$ and the approach leaves no room for any symmetry-induced collective effects. ${ }^{15,32,33}$

Recently, it was shown that PbSe QDs can be organized into ordered two dimensional (2D) superlattices in which all QDs are epitaxially connected to their nearest neighbors. ${ }^{34-38}$ As demonstrated by Evers et al., the strong electronic interdot coupling in such epitaxially connected 2D quantum-dot superlattices - epitaxial superlattices in short - results in carrier mobilities of up to $260 \mathrm{~cm}^{2} \mathrm{~V}^{-1} \mathrm{~s}^{-1}$ as deduced from THz conductivity measurements. ${ }^{39}$ This increase by two orders of magnitude attests the extensive potential of epitaxial superlattices for QD-optoelectronics. In addition, it proved possible to form epitaxial superlattices with either a square or a hexagonal, honeycomb symmetry. Theoretical investigations have pointed out that especially the honeycomb lattices feature electronic minibands with a unique structure, including a Dirac point in the centre of the miniband formed by the lowest unoccupied states of the connected QDs. ${ }^{32,40,41}$

Until now, the formation of 2D epitaxially connected $\mathrm{QD}$ superlattices has involved a meticulous procedure, where dropcasting QDs on a liquid ethylene glycol subphase results in epitaxial superlattices only in the case of specifically synthesized and purified PbSe QDs. Lacking an experimental rationale, such an approach seems prone to reproducibility and generalization issues that will slow down research on the properties and the eventual utilization of epitaxial superlattices. The development of more targeted formation protocols is bound to follow from an in-depth understanding of the surface chemistry of the nanocrystals to be connected. Most relevant in this respect is the finding that PbS and PbSe QDs synthesized using hot injection schemes tend to be cation rich, stabilized by X-type ligands such as oleates or hydroxides. ${ }^{45-47}$ Since such surface termination precludes the formation of epitaxial connections between neighboring nanocrystals, restoring stoichiometry seems a key step to achieve control over epitaxial superlattices formation. A few studies on $\mathrm{PbS}$ and CdSe QDs point in this direction. Both for PbS and CdSe QDs, initially separate QDs 
formed epitaxial connections after exposure to ammonium sulfide. ${ }^{42}$ In addition, epitaxially connected patches of PbS QDs with local square ordering could be formed by treatment with mono- and multidendate amines or by adjusting the counterion of halide salts. ${ }^{43,44}$ Although promising, the resulting structures only show local connectivity and the methods do not yield dense superlattices. This being the case, there remains a large gap between locally controlling the formation of epitaxial connections and the rational fabrication of large scale, 2D superlattices usable for applications.

Here, we build on the hypothesis that epitaxial interdot connections can only form between neighboring QDs with mutually exposed stoichiometric surfaces, which allows us to develop chemical triggers that initiate epitaxial superlattice formation. In particular, we show that monolayers of oleate-capped PbSe QDs on ethylene glycol can be turned into epitaxial superlattices by addition of sodium sulfide or butylamine, two additives known for

inducing a sulfide for oleate exchange or a lead oleate displacement, respectively. ${ }^{27,48}$ Focusing on amine addition, we find that less basic additives such as pyridine or aniline markedly promote the formation of cubic superstructures, where long range order can be imposed by a selective stripping of the lead oleate ligands prior to the initial monolayer formation. We link these observations to aniline and pyridine being weaker ligand displacing agents, as confirmed by density functional theory, and we underscore the general character of the approach developed here by not only demonstrating the formation of epitaxially connected PbSe superlattices, but also of PbS epitaxial superlattices by similar methods.

\section{Results and Discussion}

\section{Chemical Triggers for Quantum-Dot Epitaxy}

PbSe QDs were synthesized by a modification of a previously published approach, based on the injection of tri-octylphosphine-selenide (TOP-Se) into a hot solution of lead oleate under inert atmosphere. QDs made following the same recipe were used in previous stud- 

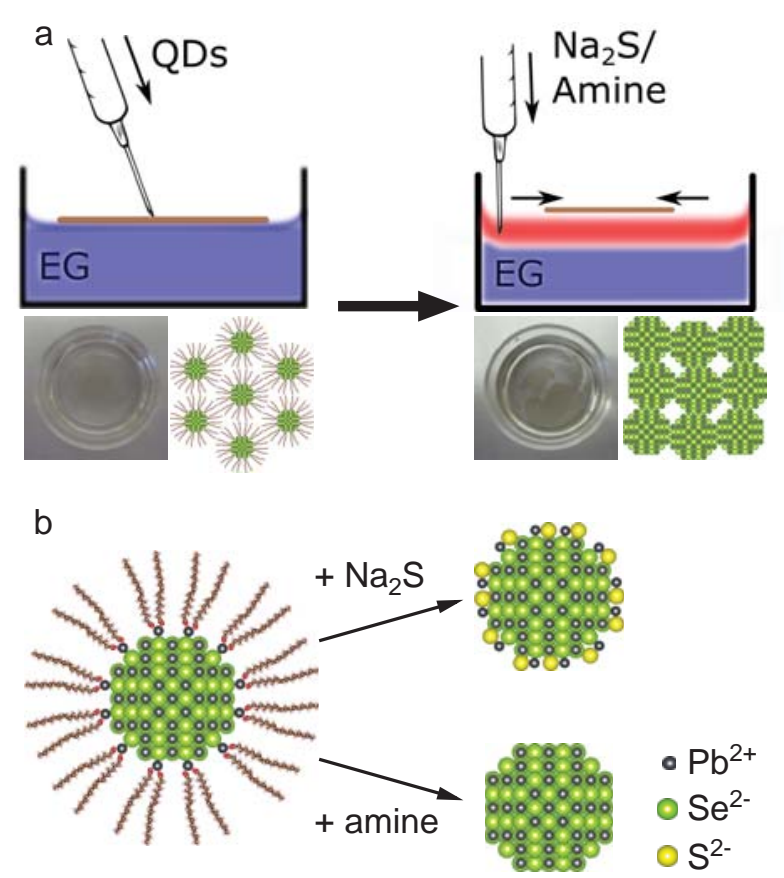

Figure 1: (a) A schematic representation of the experimental method used in this work. After dropcasting a QD monolayer, a chemical trigger that induces epitaxial connections is injected. This is seen visually as a contraction and darkening of the QD monolayer. (b) A QD crystal model showing two mechanisms to meet the requirements for QD epitaxy. $\mathrm{Na}_{2} \mathrm{~S}$ removes oleate anions and adds a stoichiometric $\mathrm{PbS}$ layer to the $\mathrm{PbSe}$ core. Alternatively, an amine removes lead oleate molecules and exposes the underlying stoichiometric PbSe facet.

ies on epitaxial superlattices of PbSe QDs. ${ }^{35,43}$ During purification however, the QDs were precipitated with acetonitrile instead of methanol as the non-solvent. This prevents spurious ligand stripping during work-up and ensures that QDs with a dense and intact ligand shell were obtained. ${ }^{49}$ Superlattices were formed by spreading as synthesized PbSe QDs on ethylene glycol at room temperature in a nitrogen-filled glovebox, where the amount of QDs was calculated so as to yield a single PbSe monolayer. Here, use of sufficiently concentrated QD dispersions proved key to form homogeneous monolayers covering the entire $4.9 \mathrm{~cm}^{2}$ ethylene glycol surface, see Figure 1a. Only in a second step, after evaporation of the solvent and formation of a self-assembled layer, the chemical trigger - typically an amine or sodium sulfide - is slowly added to the subphase. This results in a gradual change in appearance of the QD film, which visibly darkens and contracts as shown in Figure 1a. After this process 


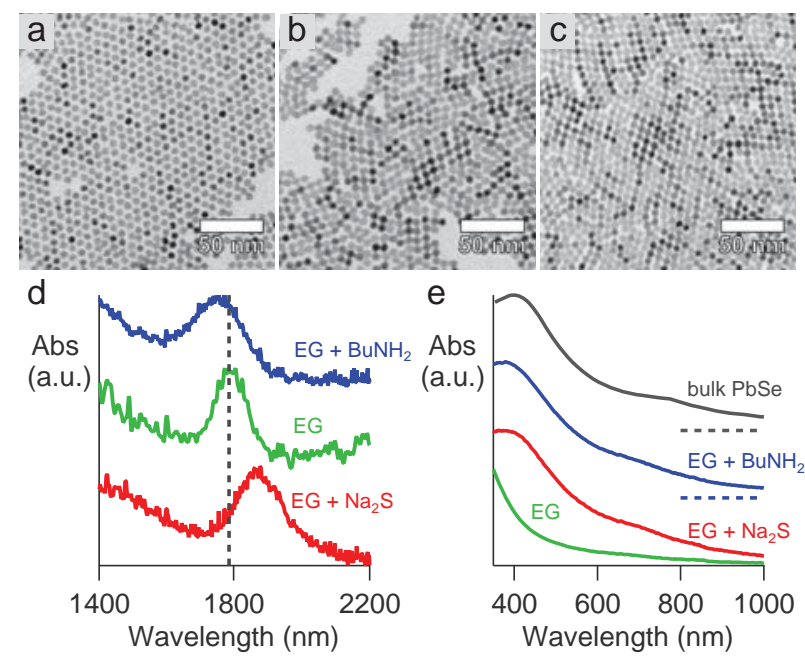

Figure 2: (a) Hexagonal, unconnected PbSe superlattice formed after spreading the QDs on ethylene glycol. (b)-(c) Superlattices showing chemically induced epitaxy after injecting $\mathrm{Na}_{2} \mathrm{~S}$ (b) or butylamine (c) into the subphase. (d) First exciton peak of superlattices formed with $\mathrm{Na}_{2} \mathrm{~S}$, butylamine or without additional reagent. (e) Same spectrum at shorter wavelengths showing the resemblance of the superlattices with the bulk PbSe absorption spectrum. The dashed lines indicate the zero-lines of the spectra.

- which takes about 5 minutes upon addition of, e.g., butylamine $\left(\mathrm{BuNH}_{2}\right)$ - the layer can be readily transferred to a substrate of choice via Langmuir-Schaefer or Langmuir-Blodgett deposition.

In first instance, we tested $\mathrm{Na}_{2} \mathrm{~S}$ and $\mathrm{BuNH}_{2}$ as two different model additives to chemically trigger the formation of epitaxial connections between neighboring quantum dots. Sulfide has been introduced before as a metal-free inorganic ligand that can transfer, for example, CdSe nanocrystals from apolar to polar solvents such as DMSO and formamide by an oleatefor-sulfide exchange. ${ }^{27,42}$ In combination with the excess $\mathrm{Pb}^{2+}$ at the $\mathrm{PbSe} \mathrm{QD}$ surface, this reaction leads to an additional, stoichiometric $\mathrm{PbS}$ layer on top of the PbSe core as shown schematically in Figure 1b. $\mathrm{BuNH}_{2}$ on the other hand is a Lewis base that was shown to strip entire metal carboxylate units from the surface of CdSe and PbS QDs by the formation of an amine-metal carboxylate complex. ${ }^{48}$ As depicted in Figure 1b, this displacement reaction removes surface lead-oleate molecules, thus exposing the underlying stoichiometric PbSe facets. 
Absent any chemical trigger, PbSe QDs spread on ethylene glycol to form a monolayer with local hexagonal ordering where the quantum-dot interdistance is determined by the originally present oleate ligands, see Figure 2a. Such monolayers can be kept stable on ethylene glycol for at least 30 minutes if $\mu \mathrm{M}$ traces of oleic acid are added to the ethylene glycol. Otherwise, transmission electron microscopy (TEM) images show local necking after 5 minutes, yet without the macroscopic contraction and darkening of the films induced by the addition of a chemical trigger. Bright field TEM images of the PbSe layers transferred after addition of a chemical trigger and visible darkening and contraction of the QD layer are shown in Figure 2b and 2c. Clearly, both $\mathrm{Na}_{2} \mathrm{~S}$ (Figure 2b) and $\mathrm{BuNH}_{2}$ (Figure 2c) addition triggers the formation of epitaxial connections between neighboring $\mathrm{PbSe}$ QDs to form a locally ordered superstructure. The idea that these chemical triggers result in either chalcogen addition or lead displacement is supported by the average QD diameter as estimated from the TEM images shown in Figure $2 \mathrm{a}-\mathrm{c}$, which decreases by $\approx 0.3 \mathrm{~nm}$ after $\mathrm{BuNH}_{2}$ addition and increases by $\approx 0.6 \mathrm{~nm}$ after $\mathrm{Na}_{2} \mathrm{~S}$ addition (see Supporting Information $\mathrm{S} 1$ ). These figures are in line with the minor blue shift and more pronounced red shift of the first exciton peak after these respective treatments, see Figure 2d and Supporting Information $\mathrm{S} 1$. In the case of amine addition, we further confirmed this point by elemental analysis using TEM-based energy dispersive X-ray spectroscopy. As shown in the Supporting Information S2, this shows that as compared to untreated films amine-treated superlattices are enriched in Se, a finding fully in line with the idea of a (partial) removal of surface $\mathrm{Pb}$ atoms by $\mathrm{BuNH}_{2}$.

As compared to the reference monolayer, the absorption spectra of the superlattices feature an absorption maximum at around $400 \mathrm{~nm}$. Such a behavior is expected for PbSe in the absence of dielectric screening, where this maximum is related to a saddle point in the dispersion relation of PbSe along the $\Delta$ direction. ${ }^{50}$ In colloidal PbSe QDs, this absorption maximum is not observed since a progressive reduction of the dielectric screening counters the drop in absorbance of bulk PbSe at shorter wavelengths. ${ }^{51}$ The presence of this absorbance 
maximum for epitaxially connected QDs indicates that in terms of dielectric screening, such a superlattice behaves more like a PbSe sheet than like isolated $\mathrm{PbSe} Q \mathrm{QD}$ in a low dielectric host, thereby attaining physical properties in between those of a PbSe thin film and colloidal PbSe QDs.

\section{Amine Basicity and Local Superlattice Symmetry}

Although both $\mathrm{Na}_{2} \mathrm{~S}$ and $\mathrm{BuNH}_{2}$ chemically trigger the epitaxial connection of neighboring PbSe QDs, we henceforth focus on metal carboxylate displacement agents such as amines as chemical triggers since these offer several advantages. First, a large variety of such triggers - typically L-type ligands - are available. Even considering amines only, they come with a wide range of basicity constants and steric profiles as monodentate or chelating ligands. This offers extensive possibilities for controlling the displacement reaction and thus the superlattice formation that is absent with small inorganic anions, where the only reaction parameter seems the concentration of sulfide or selenide anions in the subphase. Second, we expect lead carboxylate displacement to yield more homogeneous stoichiometric surfaces where the density of PbSe units is independent of the initial coverage by ligands and excess lead. In the case of an oleate-for-sulfide or selenide exchange, only excess $\mathrm{Pb}^{2+}$ ions are turned into additional $\mathrm{PbS}(\mathrm{e})$ units in the bonding crystal facet. Especially for crystal facets where Pb-oleate is weakly bound, such as the (100) facets, this can lead to patchy facets where incomplete $\mathrm{Pb}$-oleate coverage eventually leads to vacancies in the epitaxial interconnects. Finally, lead carboxylate displacement via L-type ligands can better exploit the expected difference in binding energies at different crystal facets ${ }^{52,53}$ and thus further promote long range order in the superstructures.

Figure 3 represents TEM images of epitaxial QD superlattices triggered using $\mathrm{BuNH}_{2}$ and aniline $\left(\mathrm{PhNH}_{2}\right)$, two amines with considerably different basicity constants ( $\left.K_{b}\right)$ of 3.39 and 9.42, i.e a strong and a weak base respectively. ${ }^{54,55}$ In case of $\mathrm{BuNH}_{2}$, visible darkening of the QD monolayer takes place within 5 minutes after adding $1 \mathrm{~mL}$ of a $0.1 \mathrm{M}$ 


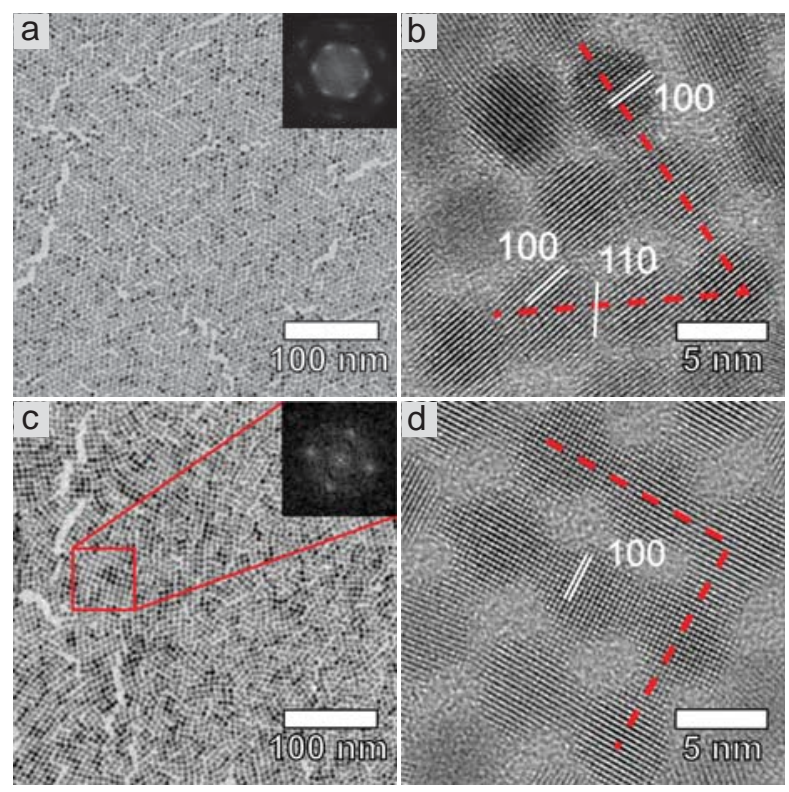

Figure 3: (a) PbSe superlattice formed with $\mathrm{BuNH}_{2}$, inset: FFT of the complete image. (b) HRTEM image showing that the superlattice in (a) is formed by a combination of epitaxial connections along the (100) and (110) facets. (c) PbSe superlattice formed with $\mathrm{PhNH}_{2}$, inset: FFT of the red square. (d) HRTEM image showing that with $\mathrm{PhNH}_{2}$ triggering the epitaxial connections occur exclusively along (100) facets.

solution to the subphase. On the other hand, after adding $1 \mathrm{~mL}$ of a $1 \mathrm{M}$ solution of $\mathrm{PhNH}_{2}$, visible darkening is delayed and only occurs after 20-30 minutes. The concentrations we use result from an initial optimization. As shown in the Supporting Information, Figure $\mathrm{S} 3$ for $\mathrm{BuNH}_{2}$, no complete superlattice is formed at lower $\mathrm{BuNH}_{2}$ concentrations, whereas the use of substantially higher concentrations leads to a network of apparently molten and recrystallized QDs. For different bases, different optimal concentrations apply in order to obtain a homogeneous, well-connected superlattice.

Comparing Figures 3a and 3c, it is clear that the different rate of superstructure formation results in superlattices with a different symmetry. $\mathrm{BuNH}_{2}$-triggered superlattices appear in essence as a strongly contracted duplicate of the originally hexagonal monolayer. More precisely, the fast Fourier transform (FFT) of the entire TEM image shown in Figure 3a (see inset) features 6 separate spots. These form the corners of a hexagon $\left(\gamma=60^{\circ}\right)$ whose width $d^{-1}$ corresponds to an interparticle distance of $5.7 \pm 0.1 \mathrm{~nm}$, a number matching the 
QD diameter of $5.76 \mathrm{~nm}$ as determined via absorption measurements. High-resolution TEM (HRTEM) imaging shows that by using $\mathrm{BuNH}_{2}$, epitaxial connections both form via (100) and (110) facets (see Figure 3b). The combination of connections through (100) and (110) facets and unconnected facets accounts for the observed long-range hexagonal ordering in the superlattice. Indeed, such an in-plane pattern cannot be formed by connecting truncated octagons through their (100) facets only. Importantly, whereas the QDs are positioned on the lattice points of a 2D hexagonal lattice, no hexagonal crystallographic motif can be discerend and local necking occurs in a random fashion. We will therefore refer to this type of structure as pseudo-hexagonal.

$\mathrm{PhNH}_{2}$-triggered superlattices on the other hand feature a patchwork of domains, that tend much more towards local square lattices. The Fourier transform of the selected domain in Figure 3c, for example, yields 4 spots forming an almost square parallellogram $\left(\gamma=85^{\circ}\right)$ whose diagonal corresponds to an interparticle distance of $5.9 \pm 0.3 \mathrm{~nm}$. Here, high-resolution TEM imaging confirms that epitaxial connections exclusively form via the (100) facets, further indicating the difference between $\mathrm{BuNH}_{2}$ and $\mathrm{PhNH}_{2}$ (Figure $3 \mathrm{~d}$ ). Importantly, the same difference in superlattice symmetry is retrieved when triggering epitaxial superlattice formation by different amines, such as pyridine $\left(\mathrm{p} K_{b}=8.83^{56}\right)$, triethylamine $\left(\mathrm{p} K_{b}=3.25^{54}\right)$ and $1,3-$ propanediamine $\left(\mathrm{p} K_{b 1}=3.74, \mathrm{p} K_{b 2}=5.30^{57}\right)$. As shown in the Supporting Information, Figure S4, all superlattices formed with a low $\mathrm{p} K_{b}$ amine show a pseudo-hexagonal geometry, while the superlattices formed with pyridine - a high $\mathrm{p} K_{b}$ amine - feature a more square-like geometry.

We addressed the amine-basicity-effect in more detail by quantitatively monitoring lead oleate displacement by amines using in-situ nuclear magnetic resonance (NMR) spectroscopy. Focusing on the resonance of the alkene protons of the oleyl chain, ${ }^{45}$ Figure 4 a shows that upon addition of $\mathrm{BuNH}_{2}$, the single broad alkene resonance recorded in the purified $\mathrm{PbSe}$ QD dispersion loses intensity whereas a second, shifted and more narrow resonance develops. Attributing the former to oleate bound to the PbSe QDs and the latter to the released lead 

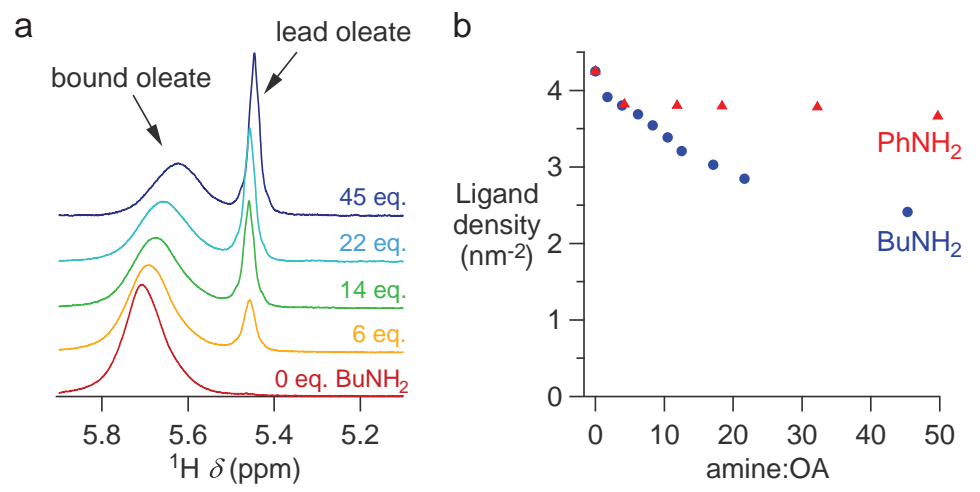

C

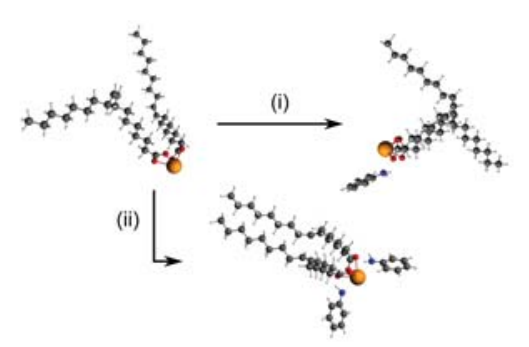

Figure 4: (a) NMR spectra of a colloidal PbSe QD solution as it is exposed to increasing amounts of butylamine. (b) The QD ligand density calculated from NMR spectra as a function of the excess amine. (c) Coordination reaction of 1 (i) and 2 (ii) aniline molecules with lead oleate, modelled using DFT calculation methods.

oleate complex, ${ }^{48,58}$ integration enables us to plot the density of bound oleate ligands as a function of the amine concentration - expressed as an equivalent of the initially present oleate - see Figure 4b. In the case of $\mathrm{BuNH}_{2}$, which we use as an example of a low $\mathrm{p} K_{b}$ amine, we find that the originally dense ligand coverage of $4.3 \mathrm{~nm}^{-2}$ drops to $3.4 \mathrm{~nm}^{-2}$ and $2.4 \mathrm{~nm}^{-2}$ upon addition of 10 and $45 \mathrm{BuNH}_{2}$ equivalents, respectively. A similar titration with $\mathrm{PhNH}_{2}$ on the other hand leads to considerably larger densities of remaining ligand at comparable equivalents. Comparing both titration curves, however, a remarkable observation is that the extent of lead oleate displacement is similar at low equivalents of $\mathrm{BuNH}_{2}$ and $\mathrm{PhNH}_{2}$. However, whereas $\mathrm{PhNH}_{2}$-displacement levels off at equivalents of 10 or more, $\mathrm{BuNH}_{2}$-displacement intensifies again around these equivalents. It thus appears that ligand displacement involves $\mathrm{Pb}$ oleate moieties with at least two markedly different binding energies to the PbSe QDs.

The difference in displacement potency between $\mathrm{BuNH}_{2}$ and $\mathrm{PhNH}_{2}$ is further confirmed by considering the coordination enthalpy, which we calculated by means of density functional theory. Depending on the reaction stoichiometry, coordination of lead oleate by $\mathrm{BuNH}_{2}$ is more exothermic by 15 to $27 \mathrm{~kJ} / \mathrm{mol}$ than coordination by $\mathrm{PhNH}_{2}$, see Table 1 and Figure 4c. Moreover, Sandeep et al. have shown that the overall ligand displacement energy strongly 
Table 1: Reaction enthalpy of the coordination of one or two amine molecules with lead oleate, calculated with at density functional theory (DFT) level of theory. (Energies given in $\mathrm{kJ} /$ mole.)

\begin{tabular}{l|c|cc|cc} 
& $\mathrm{p} K_{b}$ & $(\mathrm{i})$ & $\Delta \mathrm{E}$ & $(\mathrm{ii})$ & $\Delta \mathrm{E}$ \\
\hline Aniline & 9.42 & -62.8 & 0 & -110.2 & 0 \\
Pyridine & 8.83 & -57.8 & 5.0 & -94.7 & 15.5 \\
Butylamine & 3.39 & -78.2 & -15.4 & -136.8 & -26.6 \\
1,3-propanediamine & $3.74 / 5.30$ & -111.0 & -48.2 & -171.3 & -61.1
\end{tabular}

depends on the $\mathrm{Pb}$ oleate binding site, where displacement of excess $\mathrm{Pb}$ oleate from the (100) facets is by far the most exothermic. ${ }^{43}$ Following these authors, an interpretation of the amine-titration curves is therefore that labile $\mathrm{Pb}$ oleate ligands adsorbed to (100) facets are displaced first at low displacer equivalents, followed in the case of $\mathrm{BuNH}_{2}$ with the further displacement of more strongly bound $\mathrm{Pb}$ oleate moieties. Additionally, upon constructing a QD crystal model with cut-off planes similar to those seen in TEM images (see Supporting Information, Figure S5), we find that the (100) planes account for approximately $20 \%$ of the total surface area, which roughly coincides with the first phase of ligand displacement in the amine-titration curves where $\approx 13 \%$ of ligands are displaced. I

Interestingly, during the in-situ NMR titration with both $\mathrm{BuNH}_{2}$ and $\mathrm{PhNH}_{2}$, we found that the displacement reaction attained a steady state prior to the first NMR spectrum being measured. This time frame, however, is considerably shorter than the 20-30 minutes the PbSe QD superlattice takes to form upon addition of $\mathrm{PhNH}_{2}$. We thus conclude that lead oleate displacement is not limiting the superlattice formation rate. An alternative possibility to relate the observations that a more potent displacer results in a more rapid formation of superlattices with a pseudo-hexagonal symmetry, while a weaker displacer triggers the slow formation of square lattices, follows from considering the residual lead oleate left on the PbSe QDs by the respective displacers. Comparing the pseudo-hexagonal and square lattice, one readily sees that the more dense packing in the former makes that the remaining interparticle space leaves less room to accommodate residual ligands. Hence, the collapse of the original hexagonal monolayer in a dense pseudo-hexagonal superlattice is only possible if sufficient 

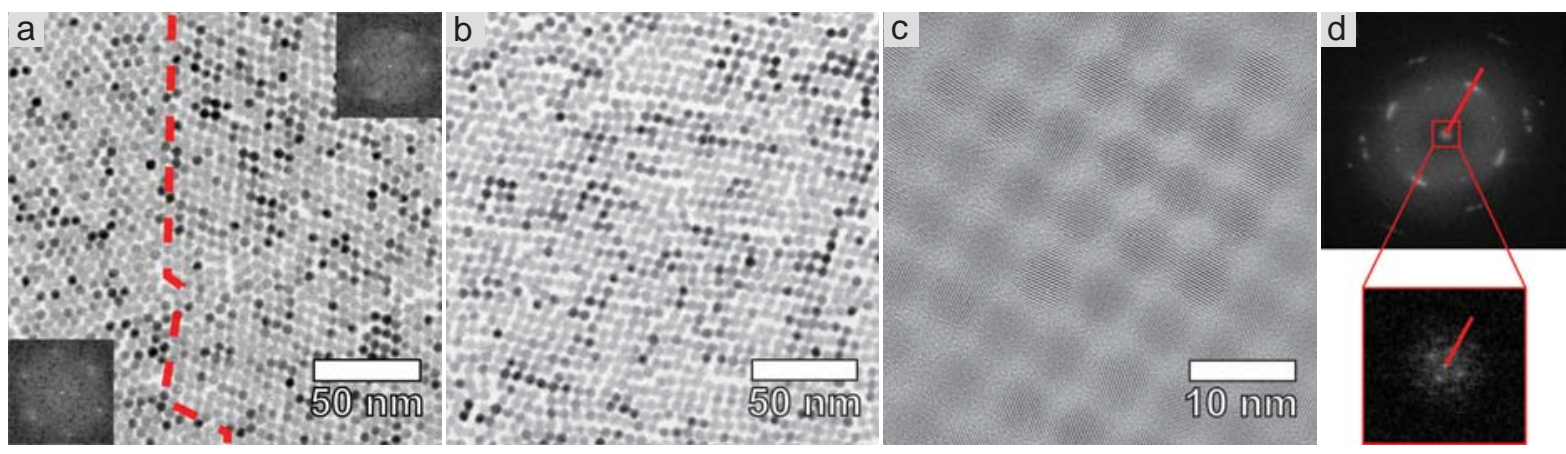

Figure 5: PbSe superlattices formed with $\mathrm{BuNH}_{2}$ (a) and $\mathrm{PhNH}_{2}$ (b) starting from $5.7 \mathrm{~nm}$ QDs with a partial ligand shell. (c) HRTEM image of a superlattice formed with $\mathrm{PhNH}_{2}$ showing the high degree of epitaxial connections. (d) FFT of (c), the zoom-in shows that the superlattice is oriented along the direction of the (100) facets.

ligands are displaced, not only from the easily stripped (100) facets ${ }^{43,52,53}$ but also from other surface facets. The NMR titration indicates that this is indeed what $\mathrm{BuNH}_{2}$ does, and what $\mathrm{PhNH}_{2}$ does not. In the case of $\mathrm{PhNH}_{2}$-triggering on the other hand, where a large fraction of the ligands remains on the surface, the formation of a close-to-square lattice can be a way to alleviate the large steric hindrance between the residual ligands on all but the (100) facets upon superlattice formation. Starting from an initially hexagonally ordered PbSe monolayer, this requires a coherent reorientation of multiple QDs, which may slows down the process and lead to the observed patchwork of differently oriented square-lattice like regions.

\section{Large Area Epitaxial Superlattices by Ligand Prestripping}

If selective ligand stripping is essential for the formation of square-like superlattices, one could expect the long range order in such superlattices to improve by a partial ligand displacement before forming the initial QD monolayer on ethylene glycol. We therefore treated an initial dispersion of $5.7 \mathrm{~nm}$ PbSe QDs with 10 equivalents of $\mathrm{BuNH}_{2}$ as ligand displacer, an equivalence slightly beyond - as we assume - the first phase of ligand displacement from the (100) surface. After purification, the solution was spread on ethylene glycol and either $1 \mathrm{~mL}$ of a $0.1 \mathrm{M} \mathrm{BuNH}_{2}$ solution or $1 \mathrm{~mL}$ of a $1 \mathrm{M} \mathrm{PhNH}_{2}$ solution was slowly injected. As indicated in Figure 5a, $\mathrm{BuNH}_{2}$ addition results in a superlattice that consists of a mixture of 

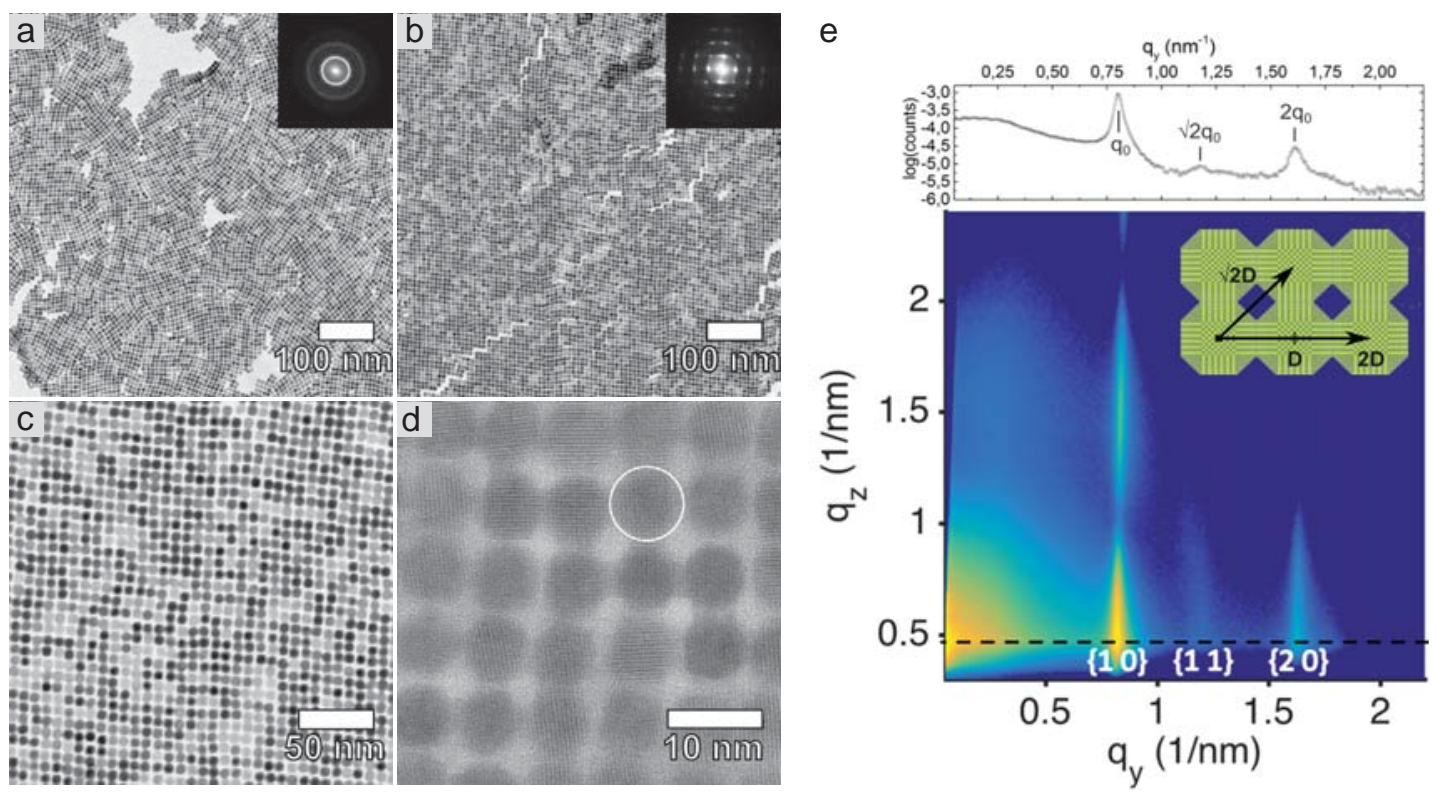

Figure 6: PbSe superlattices formed with $\mathrm{PhNH}_{2}$ starting from $7.8 \mathrm{~nm}$ QDs with a full (a) and a partial (b and c) ligand shell. (d) HRTEM image showing the high degree of epitaxial connections. The white circle represents the average particle diameter found from GISAXS measurements $(7.85 \mathrm{~nm})$ and illustrates the good agreement with TEM images. (e) GISAXS pattern of the superlattice shown in (b)-(d). The top graph shows the horizontal cut at the dashed line. The inset shows the assignment of the peaks in real space.

pseudo-hexagonal and square-like domains. However, even if square-like domains show up, large parts of the superlattice remain hexagonally ordered. $\mathrm{PhNH}_{2}$-triggering on the other hand result in an almost exclusively square-like superlattice, see Figure 5b. Moreover, we find that the combined effect of a mild ligand-displacing trigger and gentle predisplacement markedly increases the domain size. For such domains (see Figure 5c), an analysis of highresolution TEM images yields an average neck width of $2.5 \pm 0.05 \mathrm{~nm}$ and an average of 3.1 \pm 0.08 necks per QD (see Figure S6 for histograms). This last number signifies the amount of epitaxial connections formed with neighboring QDs, which for a square lattice is limited to a maximum of 4 necks per QD. Furthermore, the FFT of high-resolution TEM images not only attests the square symmetry of the lattice but also the overall coherent alignment of the (100) facets of individual PbSe QDs over the entire superlattice (see Figure 5d).

As argued before, a square-like superlattice may be formed upon triggering superlat- 
tice formation by a mild ligand displacer as the free space created between adjacent QDs enables steric hindrance between residual oleate ligands to be relaxed. In that case, the larger the QDs, the more organized the square lattice could be since the increased space left between connected QDs offers more room to reduce steric hindrance. Moreover, the shape of $\mathrm{PbSe} \mathrm{QDs}$ is known to change from quasi-spherical to truncated cubic when their size increases, which can further promote a square-like organization. The influence of the QD size is demonstrated in Figure 6, which represents TEM images of superlattices formed using $7.8 \mathrm{~nm}$ PbSe QDs. In first instance, we spread fully passivated QDs on ethylene glycol and triggered superlattice formation by addition of $1 \mathrm{~mL}$ of a $1 \mathrm{M} \mathrm{PhNH}$ solution. As shown in Figure $6 \mathrm{a}$, this results in a polycrystalline square-lattice with domain sizes of $\approx 100 \mathrm{~nm}$. The corresponding Fourier transformed image accordingly features several rings rather than a collection of separate spots. After an initial ligand displacement using 10 equivalents of $\mathrm{BuNH}_{2}$, however, the same $\mathrm{PbSe} \mathrm{QDs}$ form a square-like supercrystal after $\mathrm{PhNH}_{2}$-triggering with considerably larger domain sizes, see Figure 6b. For the example shown, the Fourier transform of the entire image is a square-like lattice of individual spots, attesting the $\mu \mathrm{m}^{2}$ ordering of the superlattice (see also Figure S7). Again, high-resolution TEM images (Figure 6d) confirm the coherent, epitaxial ordering of the PbSe QDs in the square-like lattice. The quality of the QD epitaxy in such superstructures is expressed by an average neck width of $3.5 \pm 0.06 \mathrm{~nm}$ and an average of $3.3 \pm 0.07$ necks per QD (see Figure S6).

To further demonstrate the large area homogeneity of the superlattice symmetry, we recorded a grazing incidence small angle X-ray scattering (GISAXS) pattern of a cubic PbSe QD superlattice deposited on a Si (100) substrate (Figure 6e). By taking horizontal cuts at the Si Yoneda position $\left(q_{z, S i}=0.48 \mathrm{~nm}^{-1}\right)$, this technique provides lateral information on the deposited superlattice structure averaged over the illuminated area of $\approx 39 \mathrm{~mm}^{2}$. ${ }^{59,60}$ The horizontal cut reveals three scattering maxima. The primary peak appears at a $q_{y}$ wavevector component of $0.80 \mathrm{~nm}^{-1}$, which we label as $q_{0}$. The positions of the two other side peaks then correspond to $\sqrt{2} q_{0}$ and $2 q_{0}$, as expected for the reciprocal lattice points of a cubic structure. 

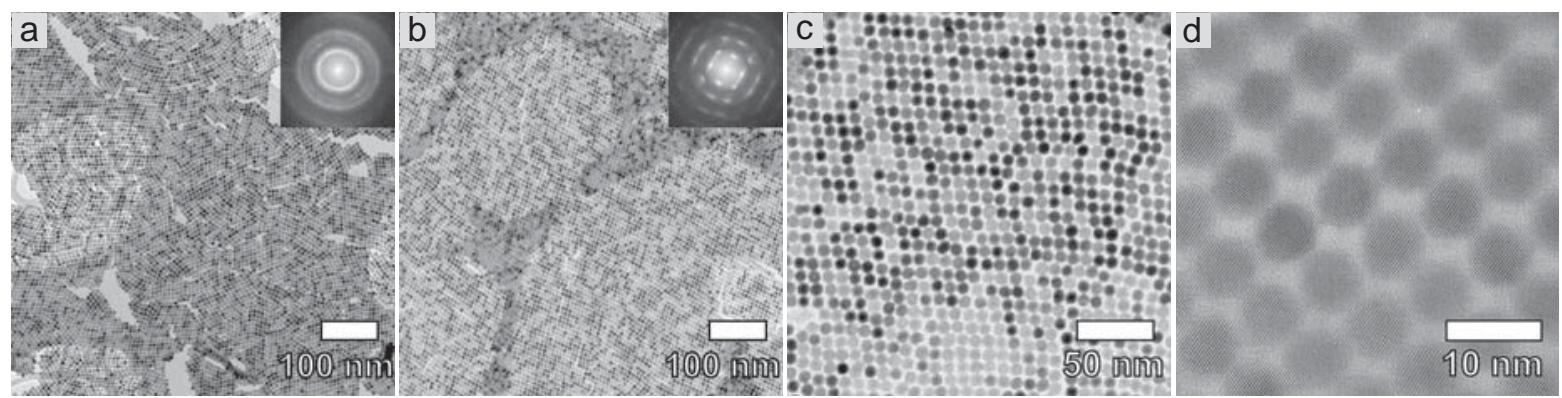

Figure 7: $\mathrm{PbS}$ superlattices formed with $\mathrm{PhNH}_{2}$ starting from $7.5 \mathrm{~nm}$ QDs with a full (a) and a partial (b and c) ligand shell. (d) HRTEM image showing the high degree of epitaxial connections.

We can thus index the three peaks as the $\{10\},\{11\}$ and $\{20\}$ reflections of a square lattice and calculate the interparticle distance along the (10) direction as $2 \pi / q_{0}=7.85 \mathrm{~nm}$. As the QDs touch each other along this direction, see Figure 6d, this distance indeed equals the QD diameter. To stress this agreement between GISAXS and TEM, we have drawn the QD diameter as determined from the GISAXS pattern as a white circle in the high-resolution TEM image (Figure 6d).

\section{Epitaxial PbS Superlattices}

As mentioned before, we presumed that an important requirement for QD epitaxy is the formation of stoichiometric crystal facets. One would thus expect that the method of chemically triggering epitaxial connections by rendering nanocrystal facets stoichiometric also applies to $\mathrm{PbS}$ QDs. To assess this point, we applied our method to $7.5 \mathrm{~nm} \mathrm{PbS}$ QDs synthesized via the approach recently published by Hendricks et al. yielding faceted, quasi-spherical QDs with a very narrow size distribution. ${ }^{61}$ Figure 7 shows PbS superlattices triggered by addition of $1 \mathrm{~mL}$ of a $1 \mathrm{M} \mathrm{PhNH}_{2}$ solution. Analogous to the case of PbSe QDs, superlattices formed with fully passivated QDs result in a polycrystalline lattice with local square ordering, resulting in concentric rings in the Fourier transformed image (Figure 7a). After reducing the ligand shell density by treating the colloidal solution with 10 equivalents of $\mathrm{BuNH}_{2}$, the QDs arrange in a distorted square-like lattice with significantly larger domains 
(Figure 7b and c). Also here, a high degree of epitaxial connections along the (100) facets is observed (Figure 7d). Analysis of high-resolution TEM images reveal an average neck width of $3.1 \pm 0.05 \mathrm{~nm}$ and an average of $3.6 \pm 0.07$ necks per QD, attesting the good quality of QD epitaxy in these superlattices. The formation of $\mathrm{PbS}$ epitaxial superlattices not only underscores the general character of our approach. It also makes the proposed method more interesting in view of device applications as $\mathrm{PbS}$ QDs are more often used than PbSe QDs for IR photodetection or solar energy conversion.

\section{Conclusion}

We demonstrate that epitaxial quantum dot superlattices can be formed using additives such as $\mathrm{Na}_{2} \mathrm{~S}$ or amines that render nanocrystal facets stoichiometric as chemical triggers. In the case of amine-triggering, we find that the extent of ligand displacement translates into superlattices with a different symmetry with the more basic, strong displacers yielding pseudo-hexagonal lattices and the less basic, mild displacers giving close to square lattices. The link between the type of amine and its ligand-displacement potency is confirmed by monitoring the displacement equilibrium using nuclear magentic resonance spectroscopy and by calculating the coordination enthalpy of the lead carboxylate complexes formed using DFT calculations. Furthermore, amines can be used to make colloidal dispersions of QDs with a reduced ligand shell density. Superlattices formed with such pre-stripped QDs show a remarkable increase of crystal domain sizes, and in combination with a mild ligand displacer such as $\mathrm{PhNH}_{2}$, we obtain almost exclusive square-like superlattices. Slightly larger QDs have a stronger tendency to form square lattices and we show that by combining QD size, ligand prestripping and amine basicity epitaxially connected square superlattices are formed with crystal domains in the $\mu \mathrm{m}^{2}$ range. Finally, we could transfer the approach to the formation of epitaxial superlattices of PbS QDs. This underscores the potential of chemically triggering superlattice formation as a rational approach to form epitaxially QD superlattices, thereby 
creating room for further improving long range order, facilitating the extension the different materials and promoting their use in QD-based opto-electronics.

\section{Methods}

PbSe QD synthesis. A stock solution of $\mathrm{Pb}$ oleate was prepared by loading $3.74 \mathrm{~g}$ of PbO (16.8 mmole) and $15.87 \mathrm{~mL}$ of oleic acid (50.3 mmole) in a three-neck flask. After flushing the flask with $N_{2}$ for 1 hour at $120^{\circ} \mathrm{C}$, the temperature was increased to $150{ }^{\circ} \mathrm{C}$ and the mixture was left to react until a clear, pale-yellow solution was obtained. The still hot solution was diluted with $67.96 \mathrm{~mL}$ of DPE and transferred to a nitrogen-filled glovebox.

TOP-Se was prepared by heating $0.7106 \mathrm{~g}$ of Se powder (9 mmole), $9.366 \mathrm{~mL}$ of trioctylphosphine $(21 \mathrm{mmole})$ and $87 \mu \mathrm{l}$ of diphenylphosphine $(0.5 \mathrm{mmole})$ to $150{ }^{\circ} \mathrm{C}$ until the Se powder was completely dissolved.

PbSe QDs with an average diameter of $5.7 \mathrm{~nm}$ were synthesized by heating $12.3 \mathrm{~mL}$ of $\mathrm{Pb}$ oleate to $180{ }^{\circ} \mathrm{C}$ and injecting $9 \mathrm{~mL}$ of TOP-Se. The temperature was set at $150{ }^{\circ} \mathrm{C}$ right before injecting the TOP-Se. After $60 \mathrm{~s}$, the reaction mixture was quenched with $15 \mathrm{~mL}$ of butanol and the synthesized QDs were precipitated by adding $15 \mathrm{~mL}$ of acetonitrile to the reaction flask. After centrifugation the supernatant was discarded and the obtained QDs were washed 2 times by dissolution in toluene, precipitation with acetonitrile and centrifugation. After the final centrifugation, the purified QDs were dissolved in $4 \mathrm{~mL}$ of toluene. All procedures were carried out under inert atmosphere to prevent oxidation of the QDs.

The size of the obtained QDs can be tuned from $3.5 \mathrm{~nm}$ to $8.5 \mathrm{~nm}$ by adjusting the injection temperature from $140{ }^{\circ} \mathrm{C}$ to $210{ }^{\circ} \mathrm{C}$, always setting the growth temperature $30{ }^{\circ} \mathrm{C}$ below the injection temperature.

Samples for NIR-UV-VIS absorption measurements were prepared by redissolving $10 \mu \mathrm{l}$ of the purified QD solution in $4 \mathrm{~mL}$ of tetrachloroethylene. 
PbS QD synthesis. PbS QDs were synthesised by a modification of a previously described method. ${ }^{61}$ In a three-neck flask, $0.2678 \mathrm{~g}$ of $\mathrm{PbO}$ (1.2 mmole), $765 \mu$ l of oleic acid (2.4 mmole) and $8.735 \mathrm{~mL}$ of n-dodecane were flushed with $N_{2}$ for $60 \mathrm{~min}$ at $120{ }^{\circ} \mathrm{C}$. After heating the mixture to $160{ }^{\circ} \mathrm{C}, 1 \mathrm{~mL}$ of a $0.8 \mathrm{M}$ N-hexyl-N'-dodecylthiourea solution in diglyme was injected. After 4 min $30 \mathrm{~s}$ the reaction mixture was cooled with a waterbath and the QDs were precipitated with $4.5 \mathrm{~mL}$ of a 1:2 acetonitrile:isopropanol mixture. After centrifugation the obtained QDs were washed 2 times by dissolution in toluene and precipitation with the same acetonitrile:isopropanol mixture.

QD solutions with a partial ligand shell. QDs with a reduced ligand shell density were obtained by reacting a colloidal QD solution with a solution containing an excess of $\mathrm{BuNH}_{2}$ compared to the total amount of oleyl chains. The number of oleyl chains were obtained from NMR measurements. Typically, $200 \mu \mathrm{l}$ of a $\mathrm{BuNH}_{2}$ solution was added to $600 \mu \mathrm{l}$ of a stirring QD solution. After reacting for about 10 minutes the mixture was purified by precipitation with acetonitrile, centrifugation and redissolution in toluene.

QD superlattices. Self-assembled QD monolayers were made by dropcasting $0.50 \mu$ of a 20-40 $\mu \mathrm{M}$ QD solution on the surface of $3.3 \mathrm{~mL}$ ethylene glycol contained in a glass vial with a diameter of $25 \mathrm{~mm}$. QD epitaxy was induced by injecting $1 \mathrm{~mL}$ of amine dissolved in ethylene glycol at a rate of $0.2 \mathrm{~mL} / \mathrm{min}$. Typical concentrations of amine solutions were 0.1 $\mathrm{M}$ for $\mathrm{BuNH}_{2}$ and $1 \mathrm{M}$ for $\mathrm{PhNH}_{2}$. The obtained superlattice was transferred to a substrate by stamping, either after reacting for $5 \min \left(\mathrm{BuNH}_{2}\right)$ or $30 \min \left(\mathrm{PhNH}_{2}\right)$. To obtain a dry film the residual ethylene glycol was removed by dipping the substrate in acetonitrile.

NMR measurements and ligand density calculation. QD suspensions were evaporated with $\mathrm{N}_{2}$ and redissolved in toluene- $d_{8}$. Nuclear Magnetic Resonance (NMR) measurements were recorded on a Bruker Avance II spectrometer operating at a ${ }^{1} \mathrm{H}$ frequency of $500.13 \mathrm{MHz}$ and equipped with a ${ }^{1} \mathrm{H},{ }^{13} \mathrm{C},{ }^{31} \mathrm{P}$ TXI-Z probe. The sample temperature was 
set to $298.2 \mathrm{~K}$ throughout. One-dimensional (1D) ${ }^{1} \mathrm{H}$ spectra were acquired using standard pulse sequences from the Bruker library. For the quantitative $1 \mathrm{D}{ }^{1} \mathrm{H}$ measurements, $64 \mathrm{k}$ time domain points were sampled with the spectral width set to $16 \mathrm{ppm}$ and a relaxation delay of $30 \mathrm{~s}$. The $1 \mathrm{D}$ spectra were multiplied with an exponential window function with a linebroadening factor of 0.3. Ligand concentrations were measured using the Digital ERETIC method implemented in Topspin 3.1.

$$
\text { ligand density }=\frac{\text { ligands } / Q D}{A_{Q D}}
$$

DFT calculations. All calculations were carried out with the computational chemistry program Turbomole, version 7.0.1. The calculations were done at density functional theory (DFT) level with the exchange-correlation functional Perdew-Burke-Ernzerhof (PBE) ${ }^{62}$ and basis set def2-SV(P). ${ }^{63,64}$ Relativistic effects were accounted for by employing the Suttgart RSC Segmented/ECP basis set with 60 core electrons on the Pb atoms. ${ }^{65}$ All structures were optimized in the gas phase.

GISAXS measurements. Samples for GISAXS measurements were prepared by stamping the PbSe QD superlattice on a clean Si (100) substrate of 10 x $15 \mathrm{~mm}$. The GISAXS experiments were performed at the DUBBLE BM26B beamline at the ESRF synchrotron, Grenoble (France). The X-ray beam energy was set at $12 \mathrm{KeV}$ by using a Si (111) doublecrystal monochromator. The beam size was $2.6 \times 0.4 \mathrm{~mm}(\mathrm{H} \times \mathrm{V})$ at the exit of the beamline. The X-ray grazing incidence angle was $0.3^{\circ}$ with respect to the plane of the sample. A continuous measurement of $60 \mathrm{~s}$ was recorded by a Pilatus $1 \mathrm{M}$ detector placed at $4174 \mathrm{~mm}$, a distance calculated by a silver behenate reference which allowed to calibrate the reciprocal q space.

TEM measurements. Transmission Electron Microscopy images were recorded on a JEOL 2200-FS operated at $200 \mathrm{kV}$. 


\section{Acknowledgement}

The authors thank Daniël Vanmaekelbergh and Wiel Evers for useful discussion. Z.H. acknowledges support by the European Comission via the Marie-Sklodowska Curie action Phonsi (H2020-MSCA-ITN-642656), the Belgian Science Policy Office (IAP 7.35, photonics@be) and IWT-Vlaanderen (SBO-MIRIS). C.D. and Z.H. acknowledge funding by BOFUGent GOA n 01G01513. J.D. acknowledges the Flemish FWO for a PhD fellowship. The GISAXS experiments were performed on beamline BM26B at the European Synchrotron Radiation Facility (ESRF), Grenoble, France. We are grateful to Daniel Hermida at the ESRF for providing assistance in using beamline BM26B, and we thank the Flemish FWO for financial support to cover the associated travel costs. I.I. would like to thank the Netherlands Organization of Scientific Research (NWO) for providing financial support within the Innovational Research Incentive (Vidi) Scheme. DFT calculations were carried out on the Dutch national e-infrastructure with the support of SURF Cooperative.

\section{Supporting Information Available}

Supporting Information shows TEM histograms of treated and untreated superlattices, the effect of $\mathrm{BuNH}_{2}$ concentration on superlattice morphology, superlattices formed using different amines, a structural PbSe nanocrystal model to estimate facet areas, EDS spectra of oleate-capped and $\mathrm{BuNH}_{2}$ treated superlattices, histograms of neck widths and

amount of necks per QD for 3 different systems and an example of a large area epitaxially connected square lattice. This material is available free of charge via the Internet at http://pubs.acs.org/.

\section{References}

1. Shevchenko, E. V.; Talapin, D. V.; Kotov, N. A.; O'Brien, S.; Murray, C. B. Structural Diversity in Binary Nanoparticle Superlattices. Nature 2006, 439, 55-9. 
2. Dong, A.; Chen, J.; Vora, P. M.; Kikkawa, J. M.; Murray, C. B. Binary Nanocrystal Superlattice Membranes Self-Assembled at the Liquid-Air Interface. Nature 2010, 466, $474-7$.

3. Sun,; Murray,; Weller,; Folks,; Moser, Monodisperse FePt Nanoparticles and Ferromagnetic FePt Nanocrystal Superlattices. Science 2000, 287, 1989-92.

4. Smith, D. K.; Goodfellow, B.; Smilgies, D. M.; Korgel, B. A. Self-Assembled Simple Hexagonal AB2 Binary Nanocrystal Superlattices: SEM, GISAXS, and Defects. J. Am. Chem. Soc. 2009, 131, 3281-3290.

5. Paik, T.; Diroll, B. T.; Kagan, C. R.; Murray, C. B. Binary and Ternary Superlattices Self-Assembled from Colloidal Nanodisks and Nanorods. J. Am. Chem. Soc. 2015, 137, 6662-6669.

6. Redl, F. X.; Cho, K.-S.; Murray, C. B.; O'Brien, S. Three-Dimensional Binary Superlattices of Magnetic Nanocrystals and Semiconductor Quantum Dots. Nature 2003, 423, $968-71$.

7. Friedrich, H.; Gommes, C. J.; Overgaag, K.; Meeldijk, J. D.; Evers, W. H.; De Nijs, B.; Boneschanscher, M. P.; De Jongh, P. E.; Verkleij, A. J.; De Jong, K. P. et al. Quantitative Structural Analysis of Binary Nanocrystal Superlattices by Electron Tomography. Nano Lett. 2009, 9, 2719-2724.

8. Evers, W. H.; Friedrich, H.; Filion, L.; Dijkstra, M.; Vanmaekelbergh, D. Observation of a Ternary Nanocrystal Superlattice and its Structural Characterization by Electron Tomography. Angew. Chem. Int. Ed. 2009, 48, 9655-9657.

9. Boneschanscher, M. P.; Evers, W. H.; Qi, W.; Meeldijk, J. D.; Dijkstra, M.; Vanmaekelbergh, D. Electron Tomography Resolves a Novel Crystal Structure in a Binary Nanocrystal Superlattice. Nano Lett. 2013, 13, 1312-1316. 
10. Evers, W. H.; Nijs, B. D.; Filion, L.; Castillo, S.; Dijkstra, M.; Vanmaekelbergh, D. Entropy-Driven Formation of Binary Semiconductor-Nanocrystal Superlattices. Nano Lett. 2010, 10, 4235-4241.

11. Eldridge, M. D.; Madden, P. a.; Frenkel, D. Entropy-Driven Formation of a Superlattice in a Hard-Sphere Binary Mixture. Nature 1993, 365, 35-37.

12. Saija, F.; Giaquinta, P. V.; Giunta, G.; Giarritta, S. P. On Entropy and Ordering in Binary Hard-Sphere Mixtures. J. Phys. Condens. Matter. 1994, 6, 9853-9865.

13. Vanmaekelbergh, D. Self-Assembly of Colloidal Nanocrystals as Route to Novel Classes of Nanostructured Materials. Nano Today 2011, 6, 419-437.

14. Geiregat, P.; Justo, Y.; Abe, S.; Flamee, S.; Hens, Z. Giant and Broad-Band Absorption Enhancement in Colloidal Quantum Dot Monolayers Through Dipolar Coupling. ACS Nano 2013, 7, 987-993.

15. Poyser, C. L.; Czerniuk, T.; Akimov, A.; Diroll, B. T.; Gaulding, E. A.; Salasyuk, A. S.; Kent, A. J.; Yakovlev, D. R.; Bayer, M.; Murray, C. B. Coherent Acoustic Phonons in Colloidal Semiconductor Nanocrystal Superlattices. ACS Nano 2016, 10, 1163-1169.

16. Wang, R. Y.; Feser, J. P.; Lee, J.-S.; Talapin, D. V.; Segalman, R.; Majumdar, A. Enhanced Thermopower in PbSe Nanocrystal Quantum Dot Superlattices. Nano Lett. 2008, 8, 2283-2288.

17. Talapin, D. V.; Murray, C. B. PbSe Nanocrystal Solids for n- and p-Channel Thin Film Field-Effect Transistors. Science 2005, 310, 86-9.

18. Liu, Y.; Tolentino, J.; Gibbs, M.; Ihly, R.; Perkins, C. L.; Liu, Y.; Crawford, N.; Hemminger, J. C.; Law, M. PbSe Quantum Dot Field-Effect Transistors with Air-Stable Electron Mobilities above 7 cm2 V1 s1. Nano Lett. 2013, 13, 1578-1587. 
19. Oh, S. J.; Berry, N. E.; Choi, J.-H.; Gaulding, E. A.; Lin, H.; Paik, T.; Diroll, B. T.; Muramoto, S.; Murray, C. B.; Kagan, C. R. Designing High-Performance PbS and PbSe Nanocrystal Electronic Devices Through Stepwise, Post-Synthesis, Colloidal Atomic Layer Deposition. Nano Lett. 2014, 14, 1559-66.

20. Tang, J.; Kemp, K. W.; Hoogland, S.; Jeong, K. S.; Liu, H.; Levina, L.; Furukawa, M.; Wang, X.; Debnath, R.; Cha, D. et al. Colloidal-Quantum-Dot Photovoltaics using Atomic-Ligand Passivation. Nat. Mater. 2011, 10, 765-71.

21. Ip, A. H.; Thon, S. M.; Hoogland, S.; Voznyy, O.; Zhitomirsky, D.; Debnath, R.; Levina, L.; Rollny, L. R.; Carey, G. H.; Fischer, A. et al. Hybrid Passivated Colloidal Quantum Dot Solids. Nat. Nanotechnol. 2012, 7, 577-82.

22. Kim, G.-H.; Garcia de Arquer, F. P.; Yoon, Y. J.; Lan, X.; Liu, M.; Voznyy, O.; Yang, Z.; Fan, F.; Ip, A. H.; Kanjanaboos, P. et al. High Efficiency Colloidal Quantum Dot Photovoltaics via Robust Self-Assembled Monolayers. Nano Lett. 2015, 15, 7691-7696.

23. Kramer, I. J.; Sargent, E. H. Colloidal Quantum Dot Photovoltaics: A Path Forward. 2011.

24. Saran, R.; Curry, R. J. Lead Sulphide Nanocrystal Photodetector Technologies. Nat. Photonics 2016, 10, 81-92.

25. Konstantatos, G.; Badioli, M.; Gaudreau, L.; Osmond, J.; Bernechea, M.; de Arquer, F. P. G.; Gatti, F.; Koppens, F. H. L. Hybrid GrapheneQuantum Dot Phototransistors with Ultrahigh Gain. Nat. Nanotechnol. 2012, 7, 363-368.

26. Konstantatos, G.; Howard, I.; Fischer, A.; Hoogland, S.; Clifford, J.; Klem, E.; Levina, L.; Sargent, E. H. Ultrasensitive Solution-Cast Quantum Dot Photodetectors. Nature 2006, 442, 180-3. 
27. Nag, A.; Kovalenko, M. V.; Lee, J.-s.; Liu, W.; Spokoyny, B.; Talapin, D. V. Metal-free Inorganic Ligands for Colloidal Nanocrystals : S2-,HS-, Se2-, HSe-,Te2-, HTe-, TeS3 2-,OH-, and NH2- as Surface Ligands. J. Am. Chem. Soc. 2011, 133, 10612-10620.

28. Gao, Y.; Aerts, M.; Sandeep, C. S. S.; Talgorn, E.; Savenije, T. J.; Kinge, S.; Siebbeles, L. D. a.; Houtepen, A. J. Photoconductivity of PbSe Quantum-Dot Solids: Dependence on Ligand Anchor Group and Length. ACS Nano 2012, 6, 9606-14.

29. Luther, J. M.; Law, M.; Song, Q.; Perkins, C. L.; Beard, M. C.; Nozik, A. J. Structural, Optical, and Electrical Properties of Self-Assembled Films of PbSe Nanocrystals Treated with 1,2-Ethanedithiol. ACS Nano 2008, 2, 271-280.

30. Yu, D.; Wang, C.; Guyot-Sionnest, P. n-Type Conducting CdSe Nanocrystal Solids. Science 2003, 300, 1277-80.

31. Dong, A.; Jiao, Y.; Milliron, D. J. Electronically Coupled Nanocrystal Superlattice Films by in situ Ligand Exchange at the Liquid-Air Interface. ACS Nano 2013, 7, 10978-84.

32. Beugeling, W.; Kalesaki, E.; Delerue, C.; Niquet, Y.-M.; Vanmaekelbergh, D.; Morais Smith, C. Topological States in Multi-Orbital HgTe Honeycomb Lattices. Nat. Commun. $\mathbf{2 0 1 5}, 6,6316$.

33. Guglietta, G. W.; Diroll, B. T.; Gaulding, E. A.; Fordham, J. L.; Li, S.; Murray, C. B.; Baxter, J. B. Lifetime, Mobility, and Diffusion of Photoexcited Carriers in LigandExchanged Lead Selenide Nanocrystal Films Measured by Time-Resolved Terahertz Spectroscopy. ACS Nano 2015, 9, 1820-1828.

34. Whitham, K.; Yang, J.; Savitzky, B. H.; Kourkoutis, L. F.; Wise, F.; Hanrath, T. Charge Transport and Localization in Atomically Coherent Quantum Dot Solids. Nat. Mater. 2016, 1-8. 
35. Evers, W. H.; Goris, B.; Bals, S.; Casavola, M.; De Graaf, J.; Roij, R. V.; Dijkstra, M.; Vanmaekelbergh, D. Low-Dimensional Semiconductor Superlattices Formed by Geometric Control over Nanocrystal Attachment. Nano Lett. 2013, 13, 2317-2323.

36. Boneschanscher, M. P.; Evers, W. H.; Geuchies, J. J.; Altantzis, T.; Goris, B.; Rabouw, F. T.; van Rossum, S. a. P.; van der Zant, H. S. J.; Siebbeles, L. D. a.; Van Tendeloo, G. et al. Long-Range Orientation and Atomic Attachment of Nanocrystals in 2D Honeycomb Superlattices. Science 2014, 344, 1377-80.

37. Williams, K. J.; Tisdale, W. A.; Leschkies, K. S.; Haugstad, G.; Norris, D. J.; Aydil, E. S.; Zhu, X. Y. Strong Electronic Coupling in Two-Dimensional Assemblies of Colloidal PbSe Quantum Dots. ACS Nano 2009, 3, 1532-1538.

38. Baumgardner, W. J.; Whitham, K.; Hanrath, T. Confined-but-Connected Quantum Solids via Controlled Ligand Displacement. Nano Lett. 2013, 13, 3225-31.

39. Evers, W. H.; Schins, J. M.; Aerts, M.; Kulkarni, A.; Capiod, P.; Berthe, M.; Grandidier, B.; Delerue, C.; Zant, H. S. J. V. D.; Overbeek, C. V. et al. High Charge Mobility in Two-Dimensional Percolative Networks of PbSe Quantum Dots Connected by Atomic Bonds. Nat. Commun. 2015, 6, 1-8.

40. Kalesaki, E.; Delerue, C.; Morais Smith, C.; Beugeling, W.; Allan, G.; Vanmaekelbergh, D. Dirac Cones, Topological Edge States, and Nontrivial Flat Bands in TwoDimensional Semiconductors with a Honeycomb Nanogeometry. Phys. Rev. X 2014, 4.

41. Delerue, C.; Vanmaekelbergh, D. Electronic Band Structure of Zinc Blende CdSe and Rock Salt PbSe Semiconductors with Silicene-Type Honeycomb Geometry. 2D Mater. 2015, 2, 034008.

42. Zhang, H.; Hu, B.; Sun, L.; Hovden, R.; Wise, F. W.; Muller, D. A.; Robinson, R. D. Sur- 
factant Ligand Removal and Rational Fabrication of Inorganically Connected Quantum Dots. Nano Lett. 2011, 11, 5356-5361.

43. Sandeep, C. S. S.; Azpiroz, J. M.; Evers, W. H.; Boehme, S. C.; Moreels, I.; Kinge, S.; Siebbeles, L. D. A.; Infante, I.; Houtepen, A. J. Epitaxially Connected PbSe QuantumDot Films : Controlled Neck Formation and Optoelectronic Properties. ACS Nano 2014, 8, 11499-511.

44. Balazs, D. M.; Dirin, D. N.; Fang, H.-H.; Protesescu, L.; ten Brink, G. H.; Kooi, B. J.; Kovalenko, M. V.; Loi, M. A. Counterion-Mediated Ligand Exchange for PbS Colloidal Quantum Dot Superlattices. ACS Nano 2015, 9, 11951-59.

45. Moreels, I.; Fritzinger, B.; Martins, J. C.; Hens, Z. Surface Chemistry of Colloidal PbSe Nanocrystals. J. Am. Chem. Soc. 2008, 130, 15081-6.

46. Petkov, V.; Moreels, I.; Hens, Z.; Ren, Y. PbSe Quantum Dots: Finite, OffStoichiometric, and Structurally Distorted. Phys. Rev. B 2010, 81, 241304.

47. Zherebetskyy, D.; Scheele, M.; Zhang, Y.; Bronstein, N.; Thompson, C.; Britt, D.; Salmeron, M.; Alivisatos, P.; Wang, L.-W. Hydroxylation of the Surface of PbS Nanocrystals Passivated with Oleic Acid. Science 2014, 344, 1380-4.

48. Anderson, N. C.; Hendricks, M. P.; Choi, J. J.; Owen, J. S. Ligand Exchange and the Stoichiometry of Metal Chalcogenide Nanocrystals: Spectroscopic Observation of Facile Metal-Carboxylate Displacement and Binding. J. Am. Chem. Soc. 2013, 135, 18536-48.

49. Hassinen, A.; Moreels, I.; De Nolf, K.; Smet, P. F.; Martins, J. C.; Hens, Z. ShortChain Alcohols Strip X-Type Ligands and Quench the Luminescence of PbSe and CdSe Quantum Dots, Acetonitrile Does Not. J. Am. Chem. Soc. 2012, 134, 20705-12.

50. Suzuki, N.; Sawai, K.; Adachi, S. Optical Properties of PbSe. J. Appl. Phys. 1995, 77, $1249-1255$. 
51. Hens, Z.; Moreels, I. Light Absorption by Colloidal Semiconductor Quantum Dots. J. Mater. Chem. 2012, 22, 10406.

52. Bealing, C. R.; Baumgardner, W. J.; Choi, J. J.; Hanrath, T.; Hennig, R. G. Predicting Nanocrystal Shape Through Consideration of Surface-Ligand Interactions. ACS Nano 2012, 6, 2118-2127.

53. Choi, J. J.; Bealing, C. R.; Bian, K.; Hughes, K. J.; Zhang, W.; Smilgies, D.-M.; Hennig, R. G.; Engstrom, J. R.; Hanrath, T. Controlling Nanocrystal Superlattice Symmetry and Shape-Anisotropic Interactions Through Variable Ligand Surface Coverage. J. Am. Chem. Soc. 2011, 133, 3131-8.

54. Vogelsong, D.; Pearson, R. Acid-Base Equilibrium Constants for 2,4-Dinitrophenol and Some Aliphatic Amines in Non-aqueous Solvents. J. Am. Chem. Soc. 1958, 80, 10381043.

55. Pankratov, A. N.; Uchaeva, I. M.; Doronin, S. Y.; Chernova, R. K. Correlations Between the Basicity and Proton Affinity of Substituted Anilines. J. Struct. Chem. 2001, 42, $739-746$.

56. Hawe, G. I.; Alkorta, I.; Popelier, P. L. A. Prediction of the Basicities of Pyridines in the Gas Phase and in Aqueous Solution. J. Chem. Inf. Model. 2010, 50, 87-96.

57. Ohtaki, H.; Tanaka, N. Ionic Equilibria in Mixed Solvents . VI . Dissociation Constants of Aliphatic Diamines in Water-Methanol Solutions. J. Phys. Chem. 1971, 75, 90-92.

58. Hens, Z.; Martins, J. C. A Solution NMR Toolbox for Characterizing the Surface Chemistry of Colloidal Nanocrystals. Chem. Mater. 2013, 25, 1211-1221.

59. Renaud, G.; Lazzari, R.; Revenant, C.; Barbier, A.; Noblet, M.; Ulrich, O.; Leroy, F.; Jupille, J.; Borensztein, Y.; Henry, C. R. et al. Real-time Monitoring of Growing Nanoparticles. Science 2003, 300, 1416-1419. 
60. Renaud, G.; Lazzari, R.; Leroy, F. Probing Surface and Interface Morphology with Grazing Incidence Small Angle X-Ray Scattering. Surf. Sci. Rep. 2009, 64, 255-380.

61. Hendricks, M. P.; Campos, M. P.; Cleveland, G. T.; Jen-La Plante, I.; Owen, J. S. A Tunable Library of Substituted Thiourea Precursors to Metal Sulfide Nanocrystals. Science 2015, 348, 1226-1230.

62. Perdew, J. P.; Burke, K.; Ernzerhof, M. Generalized Gradient Approximation Made Simple. Phys. Rev. Lett. 1996, 77, 3865-3868.

63. Eichkorn, K.; Weigend, F.; Treutler, O.; Ahlrichs, R. Auxiliary Basis Sets for Main Row Atoms and Transition Metals and Their Use to Approximate Coulomb Potentials. Theor. Chem. Acc. 1997, 97, 119-124.

64. Weigend, F.; Ahlrichs, R. Balanced Basis Sets of Split Valence, Triple Zeta Valence and Quadruple Zeta Valence Quality for H to Rn: Design and Assessment of Accuracy. Phys. Chem. Chem. Phys. 2005, 7, 3297-305.

65. Furche, F.; Ahlrichs, R.; Hättig, C.; Klopper, W.; Sierka, M.; Weigend, F. Turbomole. WIREs Comput Mol Sci 2014, 4, 91-100. 
Graphical TOC Entry

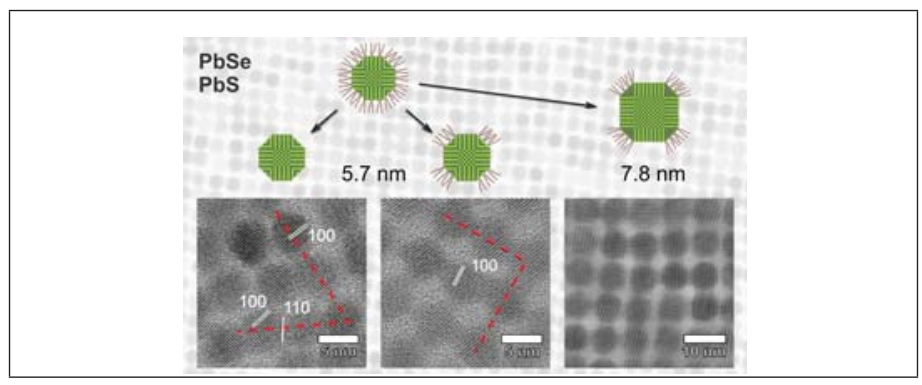

